\title{
Intelligent Reflecting Surface Operation under Predictable Receiver Mobility: A Continuous Time Propagation Model
}

\author{
Bho Matthiesen, Member, IEEE, Emil Björnson, Senior Member, IEEE, \\ Elisabeth De Carvalho, Senior Member, IEEE, and Petar Popovski, Fellow, IEEE
}

\begin{abstract}
The operation of an intelligent reflecting surface (IRS) under predictable receiver mobility is investigated. We develop a continuous time system model for multipath channels and discuss the optimal IRS configuration with respect to received power, Doppler spread, and delay spread. It is shown that the received power can be maximized without adding Doppler spread to the system. In a numerical case study, we show that an IRS having the size of just two large billboards can improve the link budget of ground to Low Earth Orbit (LEO) satellite links by up to $6 \mathrm{~dB}$. It also adds a second, almost equivalently strong, communication path that improves the link reliability.
\end{abstract}

Index Terms-Intelligent reflecting surface, reconfigurable intelligent surface, metasurface, satellite communication, Low Earth Orbit (LEO), Internet of Things (IoT), multi-objective optimization

\section{INTRODUCTION}

Intelligent reflecting surfaces (IRSs) are an emerging technology that enables tunable anomalous scattering of incident electromagnetic waves [1]-[4]. This permits active control of the propagation environment and introduces an additional optimization dimension to wireless communication networks. A main use case is range extension and recent work focuses mostly on maximizing the received power over the IRS path [5][7]. Instead, this paper considers multipath propagation with predictive receiver mobility and evaluates the implications of adding an IRS to a line-of-sight (LOS) communication scenario. This calls for the development of a continuous time model that has, to the best of the authors' knowledge, not appeared in previous works. We uncover fundamental phenomena that are not visible in the standard discrete time models.

Our motivation to consider this setup is uplink transmission of an Internet of Things (IoT) device to a satellite in Low Earth Orbit (LEO). Since the satellite's orbit is known a priori, its position is completely predictable at all times. We show

B. Matthiesen is with the Department of Communications Engineering, University of Bremen, 28359 Bremen, Germany (e-mail: matthiesen@unibremen.de). E. Björnson is with the Department of Electrical Engineering (ISY), Linköping University, 58183 Linköping, Sweden (e-mail: emil.bjornson@liu.se). E. De Carvalho and P. Popovski are with the Department of Electronic Systems, Aalborg University, 9100 Aalborg, Denmark (e-mail: \{edc, petarp\}@es.aau.dk). P. Popovski is also holder of the U Bremen Excellence Chair in the Department of Communications Engineering, University of Bremen, 28359 Bremen, Germany.

This work is supported in part by the German Research Foundation (DFG) under Germany's Excellence Strategy (EXC 2077 at University of Bremen, University Allowance), in part by ELLIIT and in part by the Danish Council for Independent Research DFF-701700271. that this information can be used at an IRS to optimize the received signal at the satellite in terms of signal-to-noise ratio (SNR), Doppler spread, or delay spread. In particular, the SNR can be maximized while simultaneously compensating Doppler spreading entirely and keeping excess delay spread due to IRS operation within one carrier signal period. Numerical results show an SNR gain of $3 \mathrm{~dB}$ to $6 \mathrm{~dB}$ for an IRS the size of two large billboards that can be achieved without introducing Doppler spread into the system. It is shown that this gain cannot be achieved by using a simple reflector of the same size and, hence, is due to the proposed optimal choice of phase shifts at the IRS. To the best of the authors knowledge, this is the first work to optimize IRS operation with predictive mobility compensation. Other application scenarios of our results include communication with vehicles on predictable paths, e.g., a car on a highway, a train, or an airplane. In the following, we first consider a generic mobility model and then, in Section IV, apply our results to the outlined satellite communication scenario.

Notation: Vectors are typeset in bold face. Euclidean points are defined as $\boldsymbol{p}=(x, y, z)^{T}$. The functions $\Re\{\cdot\},\lceil\cdot\rceil$, and $\lfloor\cdot\rfloor$ give the real value, the ceiling, and the floor of their argument, respectively. Further, $\bmod (x, y)$ is the remainder of the division of $x$ by $y$, norms are $L^{2}, j$ is the imaginary unit, $e$ is Euler's number, $\mathbb{Z}$ and $\mathbb{N}$ are the sets of integers and nonnegative numbers, respectively.

\section{System Model}

We consider an IRS in the $x y$-plane of a Cartesian coordinate system with its geometric center at the origin. It consists of $M$ columns and $N$ rows of reflecting elements placed on a rectangular grid spaced $d_{x}$ and $d_{y}$ apart. The dimensions $d_{x}$ and $d_{y}$ of each IRS element are usually within the range of $\frac{\lambda_{c}}{10}$ and $\frac{\lambda_{c}}{5}$ [8], where $\lambda_{c}$ is the carrier wavelength. The center of element $(m, n), m \in \mathcal{G}(M)$ and $n \in \mathcal{G}(N)$ with

$$
\mathcal{G}(M)=\left\{\bmod (M+1,2)-\left\lfloor\frac{M}{2}\right\rfloor, \ldots,\left\lfloor\frac{M}{2}\right\rfloor\right\},
$$

is $\boldsymbol{p}_{m, n}=\left(g\left(m, d_{x}, M\right), g\left(n, d_{y}, N\right), 0\right)$ with $g\left(m, d_{x}, M\right)=$ $m d_{x}-0.5 d_{x} \bmod (M+1,2)$. Each element has antenna gain $G\left(\theta_{m, n}, \varphi_{m, n}\right)$ with polar angle $\theta_{m, n} \in[0, \pi]$ and azimuth angle $\varphi_{m, n} \in[0,2 \pi]$ as indicated in Fig. 1. As the IRS can only receive power from one side, we assume that $G\left(\theta_{m, n}, \varphi_{m, n}\right)=0$ for $\theta_{m, n} \in\left[\frac{\pi}{2}, \pi\right]$. The singleantenna transmitter and single-antenna receiver have antenna gains $G_{\mathrm{T}}\left(\theta_{\mathrm{T}}, \varphi_{\mathrm{R}}\right)$ and $G_{\mathrm{R}}\left(\theta_{\mathrm{R}}, \varphi_{\mathrm{R}}\right)$, with $\theta_{\mathrm{T}}, \theta_{\mathrm{R}} \in[0, \pi]$ and 


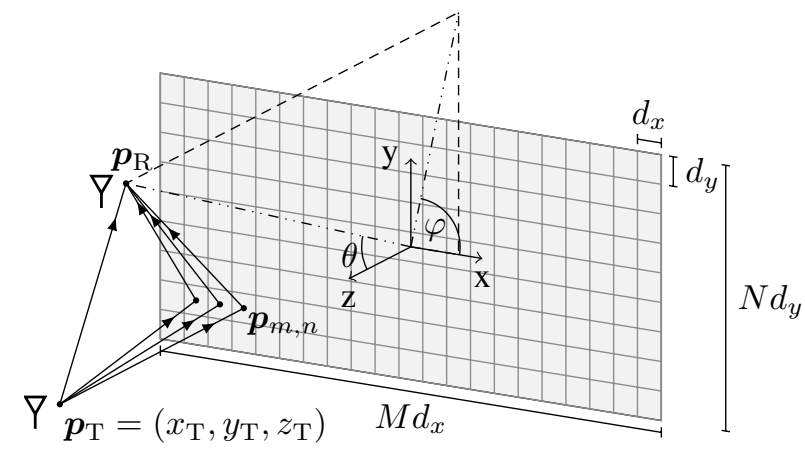

Fig. 1. Illustration of system model.

$\varphi_{\mathrm{T}}, \varphi_{\mathrm{R}} \in[0,2 \pi]$, respectively, and are located at $\boldsymbol{p}_{t}$ and $\boldsymbol{p}_{r}(t)$. The IRS and transmitter have fixed positions, while the receiver moves with time $t$. We do not consider polarization losses and coupling between the IRS elements. We also assume that all transmissions take place in the far-field. The analysis can potentially be extended to other cases and we refer to [6], [9] for more information on coupling and near-field analysis.

We consider LOS transmission between the transmitter and receiver, both over the direct path and over the IRS. ${ }^{1}$ The transmitter emits a passband signal $x_{p}(t)=\sqrt{2} \Re\left\{x(t) e^{j 2 \pi f_{c} t}\right\}$ with carrier frequency $f_{c}$. It is created from the complexbaseband signal $x(t)=x_{i}(t)+j x_{q}(t)$. The components $x_{i}(t)$ and $x_{q}(t)$ are baseband bandlimited to $B / 2$. In the absence of other propagation paths, the signal observed at an infinitesimal point at position $\boldsymbol{p}$ and time $t$ is

$$
\frac{\sqrt{G_{\mathrm{T}}\left(\theta_{\mathrm{T}}, \varphi_{\mathrm{T}}\right)}}{\sqrt{4 \pi}\left\|\boldsymbol{p}-\boldsymbol{p}_{\mathrm{T}}\right\|} x_{p}\left(t-\frac{\left\|\boldsymbol{p}-\boldsymbol{p}_{\mathrm{T}}\right\|}{c_{0}}\right),
$$

where the angles $\theta_{\mathrm{T}}$ and $\varphi_{\mathrm{T}}$ are computed between $\boldsymbol{p}_{\mathrm{T}}$ and $\boldsymbol{p}$ as $\theta_{\mathrm{T}}=\theta\left(\boldsymbol{p}_{\mathrm{T}}, \boldsymbol{p}\right)$ and $\varphi_{\mathrm{T}}=\varphi\left(\boldsymbol{p}_{\mathrm{T}}, \boldsymbol{p}\right)$ with $\theta\left(\boldsymbol{p}_{1}, \boldsymbol{p}_{2}\right)=$ $\arccos \left(\frac{z_{2}-z_{1}}{\left\|\boldsymbol{p}_{2}-\boldsymbol{p}_{1}\right\|}\right)$ and $\varphi\left(\boldsymbol{p}_{1}, \boldsymbol{p}_{2}\right)=\arctan \left(\frac{y_{2}-y_{1}}{x_{2}-x_{1}}\right)$, where the inverse tangent is defined such that it takes into account the correct quadrant for $\left(x_{2}-x_{1}, y_{2}-y_{1}\right)$.

Adding the receive antenna, which has effective area $G_{\mathrm{R}}\left(\theta\left(\boldsymbol{p}_{\mathrm{R}}(t), \boldsymbol{p}_{\mathrm{T}}\right), \varphi\left(\boldsymbol{p}_{\mathrm{R}}(t), \boldsymbol{p}_{\mathrm{T}}\right)\right) \lambda_{c}^{2} /(4 \pi)$, the observed signal (in the presence of only the direct LOS path) at the receiver is

$$
\frac{\lambda_{c} \sqrt{G_{\mathrm{T}}^{\mathrm{R}}(t) G_{\mathrm{R}}^{\mathrm{T}}(t)}}{4 \pi\left\|\boldsymbol{p}_{\mathrm{R}}(t)-\boldsymbol{p}_{\mathrm{T}}\right\|} x_{p}\left(t-\frac{\left\|\boldsymbol{p}_{\mathrm{R}}(t)-\boldsymbol{p}_{\mathrm{T}}\right\|}{c_{0}}\right)
$$

where $G_{\mathrm{T}}^{\mathrm{R}}(t)$ is the antenna gain of the transmit antenna in the direction of the receiver and $G_{\mathrm{R}}^{\mathrm{T}}(t)$ is the antenna gain of the receiver in the direction of the transmitter. These terms are computed as

$$
\begin{aligned}
& G_{\mathrm{T}}^{\mathrm{R}}(t)=G_{\mathrm{T}}\left(\theta\left(\boldsymbol{p}_{\mathrm{T}}, \boldsymbol{p}_{\mathrm{R}}(t)\right), \varphi\left(\boldsymbol{p}_{\mathrm{T}}, \boldsymbol{p}_{\mathrm{R}}(t)\right)\right), \\
& G_{\mathrm{R}}^{\mathrm{T}}(t)=G_{\mathrm{R}}\left(\theta\left(\boldsymbol{p}_{\mathrm{R}}(t), \boldsymbol{p}_{\mathrm{T}}\right), \varphi\left(\boldsymbol{p}_{\mathrm{R}}(t), \boldsymbol{p}_{\mathrm{T}}\right)\right),
\end{aligned}
$$

with $\theta$ and $\varphi$ defined as above.

The signal observed at time $t$ by the $(m, n)$ th IRS element is similar to (3) and is time-delayed by $\frac{\phi_{m, n}(t)}{2 \pi f_{c}} \geq 0$ before being scattered. This time delay is controllable in an IRS and leads to a phase shift of $\phi_{m, n}(t)$ in the emitted signal. More precisely, the re-emitted signal is

$$
\sqrt{\mu} \frac{\lambda_{c} \sqrt{G_{\mathrm{T}}^{m, n} G_{m, n}^{\mathrm{T}}}}{4 \pi\left\|\boldsymbol{p}_{m, n}-\boldsymbol{p}_{\mathrm{T}}\right\|} x_{p}\left(t-\frac{\left\|\boldsymbol{p}_{m, n}-\boldsymbol{p}_{\mathrm{T}}\right\|}{c_{0}}-\frac{\phi_{m, n}(t)}{2 \pi f_{c}}\right),
$$

${ }^{1}$ Local scattering around the transmitter is neglected for simplicity. We expect its effects to simply propagate through the IRS given that the optimization in Section III-D is done for the LOS path. As this path usually has the maximum power, this is a reasonable assumption. where the antenna gains $G_{\mathrm{T}}^{m, n}$ and $G_{m, n}^{\mathrm{T}}$ are computed in the same way as (4) and where $\mu \in[0,1]$ determines the fraction of the incident energy that is scattered. The signal that reaches the receiver over this propagation path is

$$
\begin{aligned}
\sqrt{\mu} \frac{\lambda_{c} \sqrt{G_{m, n}^{\mathrm{R}}(t) G_{\mathrm{R}}^{m, n}(t)}}{4 \pi\left\|\boldsymbol{p}_{\mathrm{R}}(t)-\boldsymbol{p}_{m, n}\right\|} \frac{\lambda_{c} \sqrt{G_{\mathrm{T}}^{m, n} G_{m, n}^{\mathrm{T}}}}{4 \pi\left\|\boldsymbol{p}_{m, n}-\boldsymbol{p}_{\mathrm{T}}\right\|} \\
\quad x_{p}\left(t-\frac{\left\|\boldsymbol{p}_{m, n}-\boldsymbol{p}_{\mathrm{T}}\right\|}{c_{0}}-\frac{\phi_{m, n}(t)}{2 \pi f_{c}}-\frac{\left\|\boldsymbol{p}_{\mathrm{R}}(t)-\boldsymbol{p}_{m, n}\right\|}{c_{0}}\right)
\end{aligned}
$$

with antenna gains $G_{m, n}^{\mathrm{R}}(t)$ and $G_{\mathrm{R}}^{m, n}(t)$ computetd as in (4).

Assuming the propagation channel consists of the direct path and the scattered paths via the IRS, we obtain the received passband signal as

$$
\begin{aligned}
y_{p}(t) & =A_{0}(t) x_{p}\left(t-\tau_{0}(t)\right) \\
& +\sum_{m, n} A_{m, n}(t) x_{p}\left(t-\tau_{m, n}(t)-\frac{\phi_{m, n}(t)}{2 \pi f_{c}}\right)+n_{p}(t)
\end{aligned}
$$

with $n_{p}(t)$ being a white Gaussian noise process with power spectral density $N_{0}$. The amplitude gains and delays are

$$
A_{0}(t)=\frac{\lambda_{c} \sqrt{G_{\mathrm{T}}^{\mathrm{R}}(t) G_{\mathrm{R}}^{\mathrm{T}}(t)}}{4 \pi\left\|\boldsymbol{p}_{\mathrm{R}}(t)-\boldsymbol{p}_{\mathrm{T}}\right\|}, \quad \tau_{0}(t)=\frac{\left\|\boldsymbol{p}_{\mathrm{R}}(t)-\boldsymbol{p}_{\mathrm{T}}\right\|}{c_{0}},
$$

for the direct path and

$$
\begin{aligned}
A_{m, n}(t) & =\sqrt{\mu} \frac{\lambda_{c}^{2}}{16 \pi^{2}} \frac{\sqrt{G_{m, n}^{\mathrm{R}}(t) G_{\mathrm{R}}^{m, n}(t) G_{\mathrm{T}}^{m, n} G_{m, n}^{\mathrm{T}}}}{\left\|\boldsymbol{p}_{\mathrm{R}}(t)-\boldsymbol{p}_{m, n}\right\|\left\|\boldsymbol{p}_{m, n}-\boldsymbol{p}_{\mathrm{T}}\right\|}, \\
\tau_{m, n}(t) & =\frac{\left\|\boldsymbol{p}_{m, n}-\boldsymbol{p}_{\mathrm{T}}\right\|+\left\|\boldsymbol{p}_{\mathrm{R}}(t)-\boldsymbol{p}_{m, n}\right\|}{c_{0}}
\end{aligned}
$$

for the path over the $(m, n)$ th IRS element. Observe that the tunable delay $\frac{\phi_{m, n}(t)}{2 \pi f_{c}}$ of the IRS is not included in $\tau_{m, n}(t)$ as it can be directly controlled to optimize the propagation environment. The corresponding complex baseband signal is

$$
\begin{aligned}
& y(t)=A_{0}(t) e^{-j 2 \pi f_{c} \tau_{0}(t)} x\left(t-\tau_{0}(t)\right) \\
& +\sum_{m, n} A_{m, n}(t) e^{-j 2 \pi f_{c} \tau_{m, n}(t)-j \phi_{m, n}(t)} \\
& \quad x\left(t-\tau_{m, n}(t)-\frac{\phi_{m, n}(t)}{2 \pi f_{c}}\right)+n(t) .
\end{aligned}
$$

It can be observed that the complex pseudo-baseband channel response $^{2}$ of the $(m, n)$ th IRS path is

$$
A_{m, n}(t) e^{-j 2 \pi f_{c} \tau_{m, n}(t)} e^{-j \phi_{m, n}(t)} .
$$

This matches with the usual narrow-band model of IRS communication, e.g., used in [3], [5], [7], [8] and others, where the IRS operation is represented by a multiplicative coefficient $\sqrt{\mu} e^{j \gamma_{m, n}}$. Observe that causality requires $\gamma_{m, n}$ to be negative as opposed to the usual assumption of $\gamma_{m, n} \geq 0$.

\section{OPTIMIZING THE IRS OPERATION}

In the considered setup, the sole purpose of the IRS is to improve the channel between the transmitter and the receiver. In the described LOS propagation environment, the direct channel is subject to a transmission delay and a Doppler shift. Introducing the IRS turns this channel into a multipath environment with the usual positive effects of increased received power and transmission diversity. It also potentially

\footnotetext{
${ }^{2}$ The complex baseband channel response is bandlimited and obtained by low-pass filtering (12).
} 
introduces delay spread and Doppler spread which necessitates more complex receivers and might degrade the performance. As the adjustable phase shifts of the IRS can be configured in almost arbitrary ways, any of these multi-path effects can be either amplified or attenuated. We will first independently optimize the phase shifts with respect to maximum received power, minimum Doppler spread, and minimum delay spread. Then, we discuss trade-offs between these scalar solutions and discuss the joint optimization with respect to these metrics, using the multi-objective optimization methodology [10], [11].

\section{A. Received Power}

Assume the transmitter is sending a constant signal with power $P_{\mathrm{T}}$. Then, the average received power in a time interval of length $2 T$ centered around time instance $t_{0}$ is

$$
\frac{1}{2 T} \int_{t_{0}-T}^{t_{0}+T}|y(t)-n(t)|^{2} d t=\frac{1}{2 T} \int_{t_{0}-T}^{t_{0}+T} P_{\mathrm{R}}(t) d t,
$$

where $P_{\mathrm{R}}(t)$ is the instantaneous receive power

$$
P_{\mathrm{T}}\left|A_{0}(t) e^{-j 2 \pi f_{c} \tau_{0}(t)}+\sum_{m, n} A_{m, n}(t) e^{-j 2 \pi f_{c} \tau_{m, n}(t)-j \phi_{m, n}(t)}\right|^{2} .
$$

Due to the monotonicity of integration [12, Thm 12.4], the average received power is maximized if $P_{\mathrm{R}}(t)$ is maximized at every $t$. It follows from the triangle inequality that this requires all the terms to have the same phase [5], [8], i.e.,

$$
2 \pi f_{c} \tau_{0}(t)=2 \pi f_{c} \tau_{m, n}(t)+\phi_{m, n}(t)-2 \pi k_{m, n}(t)
$$

for all $m, n$ and arbitrary $k_{m, n}(t) \in \mathbb{Z}$, where $k_{m, n}(t)$ are additional full carrier signal period delays that do not affect the received power. Hence, choosing

$$
\phi_{m, n}(t)=2 \pi f_{c}\left(\tau_{0}(t)-\tau_{m, n}(t)\right)+2 \pi k_{m, n}(t)
$$

maximizes the receive SNR. This resembles the result in previous works [5], [8] where it is obtained from discrete time models. The causality requirement $\phi_{m, n}(t) \geq 0$ implies

$$
k_{m, n}(t) \geq f_{c}\left(\tau_{m, n}(t)-\tau_{0}(t)\right) .
$$

It follows from the triangle inequality that

$$
\tau_{m, n}(t) \geq \frac{\left\|\boldsymbol{p}_{m, n}-\boldsymbol{p}_{\mathrm{T}}+\boldsymbol{p}_{\mathrm{R}}(t)-\boldsymbol{p}_{m, n}\right\|}{c_{0}}=\tau_{0}(t)
$$

for all $m, n$. Hence, $k_{m, n}(t) \in \mathbb{N}$ and, unless $\tau_{m, n}(t)=\tau_{0}(t)$, $k_{m, n}(t) \geq 1$.

\section{B. Doppler Spread}

The Doppler spread is the maximum difference in instantaneous frequency over all significant propagation paths, i.e., $D_{\mathrm{s}}(t)=\max \left\{D_{\mathrm{s}, 0}(t), D_{\mathrm{s}, \mathrm{IRS}}(t)\right\}$ where

$$
D_{\mathrm{s}, 0}(t)=f_{c} \max _{m, n}\left|\frac{d}{d t}\left(\tau_{m, n}(t)+\frac{\phi_{m, n}(t)}{2 \pi f_{c}}\right)-\frac{d}{d t} \tau_{0}(t)\right|
$$

is the Doppler spread between the direct path and the IRS, and

$$
\begin{aligned}
D_{\mathrm{s}, \mathrm{IRS}}(t)=f_{c} \max _{m, n, m^{\prime}, n^{\prime}} & \frac{d}{d t}\left(\tau_{m, n}(t)+\frac{\phi_{m, n}(t)}{2 \pi f_{c}}\right) \\
& -\frac{d}{d t}\left(\tau_{m^{\prime}, n^{\prime}}(t)+\frac{\phi_{m^{\prime}, n^{\prime}}(t)}{2 \pi f_{c}}\right) \mid
\end{aligned}
$$

is the Doppler spread across the IRS. Clearly, $D_{\mathrm{s}, 0}(t)$ is minimized if

$$
\frac{d}{d t} \phi_{m, n}(t)=2 \pi f_{c} \frac{d}{d t}\left(\tau_{0}(t)-\tau_{m, n}(t)\right)
$$

for all $m, n$. Then, $D_{\mathrm{s}, 0}(t)=0$ and

$$
\begin{gathered}
\frac{d}{d t}\left(\tau_{m, n}(t)+\frac{\phi_{m, n}(t)}{2 \pi f_{c}}\right)-\frac{d}{d t}\left(\tau_{m^{\prime}, n^{\prime}}(t)+\frac{\phi_{m^{\prime}, n^{\prime}}(t)}{2 \pi f_{c}}\right) \\
=\frac{d}{d t} \tau_{m, n}(t)+\frac{d}{d t}\left(\tau_{0}(t)-\tau_{m, n}(t)\right) \\
-\frac{d}{d t} \tau_{m^{\prime}, n^{\prime}}(t)-\frac{d}{d t}\left(\tau_{0}(t)-\tau_{m^{\prime}, n^{\prime}}(t)\right)=0
\end{gathered}
$$

Hence, (21) also minimizes $D_{\mathrm{s}, \mathrm{IRS}}(t)$ and the Doppler spread is zero. From a physical perspective, this choice of $\phi_{m, n}(t)$ compensates for the difference in relative velocities between the direct path and the IRS as observed by the receiver. While this approach does not remove the Doppler shift due to the movement of the receiver, it prevents the introduction of additional frequency components in the IRS paths.

\section{Delay Spread}

The delay spread is the maximum difference in propagation time over all significant transmission paths, i.e., $T_{\mathrm{d}}(t)=$ $\max \left\{T_{\mathrm{d}, 0}, T_{\mathrm{d}, \mathrm{IRS}}\right\}$ where

$$
T_{\mathrm{d}, 0}(t)=\max _{m, n}\left\{\tau_{m, n}(t)+\frac{\phi_{m, n}(t)}{2 \pi f_{c}}\right\}-\tau_{0}(t)
$$

is the delay spread between the IRS and direct path, and

$$
\begin{aligned}
T_{\mathrm{d}, \mathrm{IRS}}(t)=\max _{m, n}\left\{\tau_{m, n}(t)\right. & \left.+\frac{\phi_{m, n}(t)}{2 \pi f_{c}}\right\} \\
& -\min _{m, n}\left\{\tau_{m, n}(t)+\frac{\phi_{m, n}(t)}{2 \pi f_{c}}\right\}
\end{aligned}
$$

is the delay spread of the IRS.

Since $\phi_{m, n}(t) \geq 0$ and due to (18),

$$
\min _{m, n}\left\{\tau_{m, n}(t)+\frac{\phi_{m, n}(t)}{2 \pi f_{c}}\right\} \geq \min _{m, n}\left\{\tau_{m, n}(t)\right\} \geq \tau_{0}(t) .
$$

Hence, $T_{\mathrm{d}, \mathrm{IRS}}(t) \leq \max _{m, n}\left\{\tau_{m, n}(t)+\frac{\phi_{m, n}(t)}{2 \pi f_{c}}\right\}-\tau_{0}(t)=$ $T_{\mathrm{d}, 0}(t)$ and the delay spread simplifies to

$$
T_{\mathrm{d}}(t)=T_{\mathrm{d}, 0}(t)=\max _{m, n}\left\{\tau_{m, n}(t)+\frac{\phi_{m, n}(t)}{2 \pi f_{c}}\right\}-\tau_{0}(t) .
$$

Thus, the delay spread is an increasing function in $\phi_{m, n}(t)$ and choosing it as small as possible reduces the delay spread. In particular, the unique solution of minimizing $T_{\mathrm{d}}(t)$ is $\phi_{m, n}(t)=0$ for all $m, n$ and $t$.

\section{Pareto Optimal Phase Shifts}

Our design goal is to simultaneously maximize the received power and minimize delay and Doppler spread, i.e., find a solution to the multi-objective optimization problem

$$
\max _{\forall m, n: \phi_{m, n}(t)}\left[P_{\mathrm{R}}(t)-D_{\mathrm{s}}(t)-T_{\mathrm{d}}(t)\right] .
$$

It can be seen from (16) and (27) that jointly maximizing $P_{\mathrm{R}}(t)$ and minimizing $T_{\mathrm{d}}(t)$ is impossible. Hence, (28) has no single solution but, instead, an infinite number of noninferior solutions [10], [11]. A vector $\left(\phi_{m, n}(t)\right)_{m, n}$ is considered a solution to (28) if it achieves an objective vector where no component can be improved without worsening at least one other objective. Such a point is called a Pareto optimal solution and the set of all such vectors is the Pareto optimal solution set of (28). Selecting an appropriate solution from this Pareto 
set is, in general, no trivial task. However, in this case it is easy to make a strong argument for a particular solution.

Clearly, maximum received power is the most important among the objectives in (28) to ensure good reception as it maximizes the SNR. Moreover, as we will show in the sequel, $P_{\mathrm{R}}(t)$ and $-D_{\mathrm{S}}(t)$ can be maximized simultaneously, and the relative increase in $T_{\mathrm{d}}(t)$ can be kept small when maximizing the other two objectives. These aspects suggest lexicographic ordering $[13, \S 4.2]$ as the solution strategy, where the objectives in (28) are ordered by their absolute importance and maximized successively. In particular, consider the lexicographic order

$$
P_{\mathrm{R}}(t) \succ-D_{\mathrm{s}}(t) \succ-T_{\mathrm{d}}(t),
$$

where " $\succ$ " stands for "is more important than", and let $\mathcal{P}_{\mathrm{R}}(t)$ be the solution set of maximizing $P_{\mathrm{R}}(t)$, i.e., all solutions that satisfy (16). Then, the lexicographic solution (29) of (28) is obtained by first refining $\mathcal{P}_{\mathrm{R}}(t)$ such that it only contains solutions that minimize $D_{\mathrm{s}}(t)$ over $\mathcal{P}_{\mathrm{R}}(t)$ and then selecting a solution from this set that minimizes $T_{\mathrm{d}}(t)$.

Theorem 1: The lexicographic solution (29) of (28) is

$$
\phi_{m, n}(t)=2 \pi \bmod \left(f_{c}\left(\tau_{0}(t)-\tau_{m, n}(t)\right), 1\right) .
$$

It is a Pareto optimal solution of (28) and results in an instantaneous received power

$$
P_{\mathrm{R}}(t)=P_{\mathrm{T}}\left|A_{0}(t)+\sum_{m, n} A_{m, n}(t)\right|^{2},
$$

Doppler spread $D_{\mathrm{S}}(t)=0$, and delay spread

$$
T_{\mathrm{d}}(t)=\frac{1}{f_{c}} \max _{m, n}\left\lceil f_{c}\left(\tau_{m, n}(t)-\tau_{0}(t)\right)\right\rceil .
$$

Proof: The received power is maximized for all phase shifts that satisfy (16). The derivative of (16) is

$$
\frac{d}{d t} \phi_{m, n}(t)=2 \pi f_{c} \frac{d}{d t}\left(\tau_{0}(t)-\tau_{m, n}(t)\right)+2 \pi \frac{d}{d t} k_{m, n}(t) .
$$

Except for the last term, this is equivalent to the optimality condition for $\min D_{\mathrm{s}}(t)$ in (21). $k_{m, n}(t)$ is a step function $k_{m, n}(t)=\sum_{i=1}^{n} \alpha_{i} H\left(t-t_{i}\right)$ where $H(t)$ is the Heaviside step function and $\alpha_{i} \in\{-1,1\}$. Its derivative is $\frac{d}{d t} k_{m, n}(t)=$ $\sum_{i=1}^{n} \alpha_{i} \delta\left(t-t_{i}\right)$ with $\delta(t)$ being the Dirac delta function Thus, the derivative of $k_{m, n}(t)$ vanishes except for the time instants where $k_{m, n}(t)$ changes its integer value. ${ }^{3}$ However, since every change in $k_{m, n}(t)$ results in a $2 \pi$ phase shift, it does not lead to discontinuities in the signal and, hence, has no impact on the instantaneous frequency. Therefore, $\frac{d}{d t} k_{m, n}(t)$ can be regarded as effectively zero and (16) minimizes the Doppler shift. From (27), the delay spread for (16) is

$$
\begin{aligned}
& T_{\mathrm{d}}(t)+\tau_{0}(t) \\
= & \max _{m, n}\left\{\tau_{m, n}(t)+\frac{2 \pi f_{c}\left(\tau_{0}(t)-\tau_{m, n}(t)\right)+2 \pi k_{m, n}(t)}{2 \pi f_{c}}\right\} \\
= & \max _{m, n}\left\{\tau_{m, n}(t)+\tau_{0}(t)-\tau_{m, n}(t)+\frac{k_{m, n}(t)}{f_{c}}\right\}
\end{aligned}
$$

and, hence, $T_{\mathrm{d}}(t)=\max _{m, n}\left\{k_{m, n}(t)\right\} / f_{c}$. Thus, $k_{m, n}(t)$ should be chosen as small as possible to minimize the delay spread. Thus, the optimal $k_{m, n}(t)$ is, due to causality and (17), $k_{m, n}(t)=\left\lceil f_{c}\left(\tau_{m, n}(t)-\tau_{0}(t)\right)\right\rceil$. Then,

$$
\begin{aligned}
\phi_{m, n}(t) & =2 \pi f_{c}\left(\tau_{0}(t)-\tau_{m, n}(t)\right)+2 \pi\left\lceil f_{c}\left(\tau_{m, n}(t)-\tau_{0}(t)\right)\right\rceil \\
& =2 \pi \bmod \left(f_{c}\left(\tau_{0}(t)-\tau_{m, n}(t)\right), 1\right) .
\end{aligned}
$$

${ }^{3}$ This cannot be prevented since a practical IRS is only capable of implementing phase shifts in the order of a few multiples of $2 \pi$ [7, §V-B].

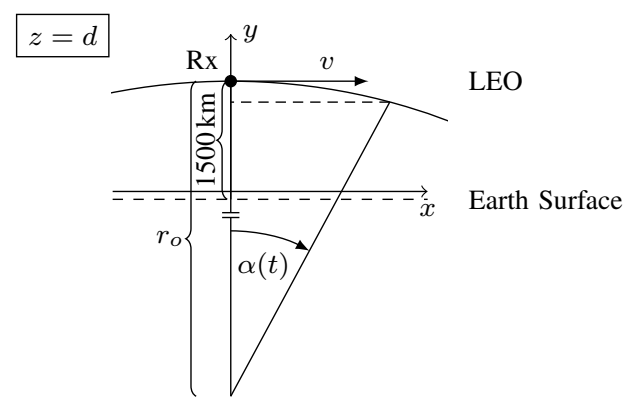

Fig. 2. Orbital plane of the satellite and trajectory computation (not to scale).

This solution is Pareto optimal due to [13, Thm. 4.2.1].

Comparing (32) to (27), it can be observed that $T_{\mathrm{d}}(t)$ is increased by at most $\frac{1}{f_{c}}$ over its physical minimum, which is extremely small compared to the overall delay (spread). Hence, the solution in Theorem 1 jointly optimizes two out of three performance metrics and is very close to the optimal solution of the third. Instead, reversing the lexicographic order in (29) leads to a slightly smaller delay spread but much larger Doppler spread and no apparent gain of the IRS. Changing the order of $P_{\mathrm{R}}(t)$ and $-D_{\mathrm{S}}(t)$ in (29) results in the same solution.

\section{Numerical EVAluation}

We consider the uplink transmission of a fixed ground terminal, e.g., an IoT device, to a satellite in LEO at an altitude of $1500 \mathrm{~km}$. This scenario is described as Deployment-D3 in [14]. The communication takes place in the S-band at a carrier frequency of $f_{c}=2 \mathrm{GHz}$ and requires a minimum elevation angle between ground terminal and satellite of $10^{\circ}$. The transmitter is located on the ground with a horizontal distance of $1 \mathrm{~km}$ to the IRS center, while the IRS has an elevation above ground of $100 \mathrm{~m}$. Hence, $p_{\mathrm{T}}=(0,-100 \mathrm{~m}, 1 \mathrm{~km})$. The receiver is moving parallel to the $x y$-plane at a horizontal distance $d$ to the IRS and in an ideal Keplerian circular orbit. The Earth is assumed as a perfect sphere with radius $6371 \mathrm{~km}$. Thus, the orbital radius of the receiver is $r_{o}=7871 \mathrm{~km}$ and the orbital velocity is $v=\sqrt{\frac{\mu}{r_{o}}}$ with $\mu$ being Kepler's constant defined as $\mu=3.986004 \times 10^{5} \mathrm{~km}^{3} / \mathrm{s}^{2}$ [15]. The computation of the trajectory $p_{\mathrm{R}}(t)$ is illustrated in Fig. 2. In particular, the angle $\alpha(t)$ is obtained from the orbital period $\frac{2 \pi r_{o}}{v}$ as $\alpha(t)=\frac{v}{r_{o}} t=\sqrt{\frac{\mu}{r_{o}^{3}}} t$. Assuming the satellite has position $p_{\mathrm{R}}(0)=(0,1499.9 \mathrm{~km}, d)$ at time $t=0$, its trajectory is computed as $p_{\mathrm{R}}(t)-p_{\mathrm{R}}(0)=\left(r_{o} \sin (\alpha(t)), r_{o}(\cos (\alpha(t))-1), 0\right)$.

The IRS has dimensions $18.3 \mathrm{~m} \times 12.2 \mathrm{~m}$, which corresponds to the size of two large US billboards. With an element size of $d_{x}=d_{y}=\frac{\lambda_{c}}{5}$, this amounts to $610 \times 407$ elements that are modeled as lossless diffuse reflectors $(\mu=1)$ with the planar antenna gain pattern $G(\theta, \varphi)=\frac{4 \pi}{\lambda_{c}^{2}} d_{x} d_{y} \cos (\theta)$ for $\theta \in\left[0, \frac{\pi}{2}\right]$ and zero otherwise. With phase shifts as in (30), the channel gain is given as $\frac{P_{\mathrm{R}}(t)}{P_{\mathrm{T}}}$ with $P_{\mathrm{R}}(t)$ as in (31).

Assuming unobstructed view, no atmospheric effects, and isotropic transmit and receive antennas, i.e., $G_{\mathrm{T}}(\theta, \varphi)=$ $G_{\mathrm{R}}(\theta, \varphi)=1$, the channel gain is displayed in Fig. 3. As baseline scheme, we compare to the case without IRS, i.e., $A_{m, n}(t)=0$ for all $m, n$ in (31), and to scenarios where the IRS is configured to approximate a specular reflector 


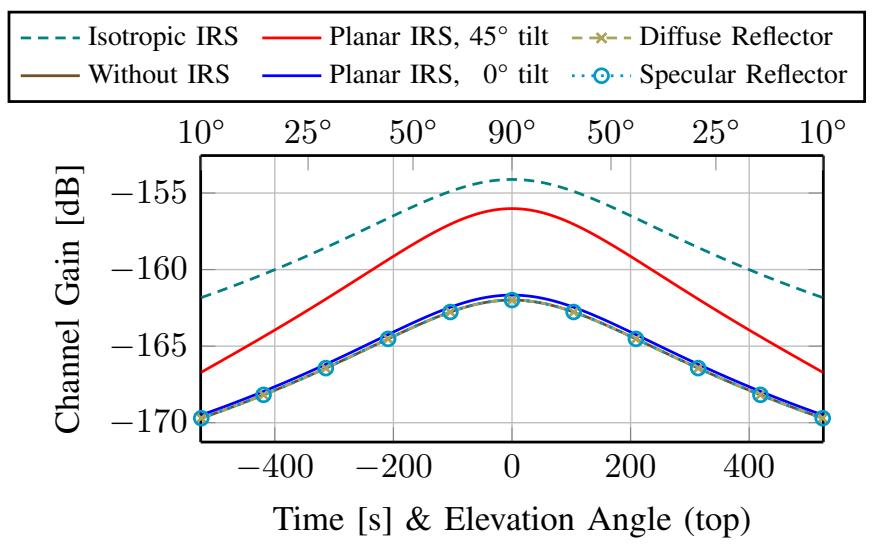

Fig. 3. Channel gain over time for one satellite pass. The plots of the scenarios without IRS and with reflectors are congruent.

and as a diffuse reflector with phase shifts chosen by Snell's law and uniformly in the interval $[0,2 \pi]$, respectively. These configurations emulate the behavior of a planar obstacle that reflects the signal in place of the IRS. Further, to obtain an upper bound that serves as a best case deployment, we consider an IRS with isotropic elements, i.e., $G(\theta, \varphi)=1$ for $\theta \in\left[0, \frac{\pi}{2}\right]$ and zero otherwise. The number of elements in this case is chosen such that the effective antenna area matches the size of the IRS. With an effective area per element of $\frac{\lambda^{2}}{4 \pi}$ this amounts to $433 \times 288$ elements.

It can be observed from Fig. 3 that the gain of the IRS with isotropic elements over the baseline is $7.9 \mathrm{~dB}$. This is also directly displayed in Fig. 4. In contrast, the gain of the IRS with planar elements is negligible. This is due to the unfavorable angle of the receiver towards the IRS which reduces the effective area of the IRS to nearly zero. This issue can be avoided by physically rotating the IRS towards the sky. In particular, by rotating the $x$-axis by $45^{\circ}$ and keeping the IRS in the $x y$-plane an uptilt of $45^{\circ}$ is achieved. This results in much better performance as can be observed from Figs. 3 and 4 . Depending on the elevation angle, the gain is between $3 \mathrm{~dB}$ and $5.97 \mathrm{~dB}$ over the baseline. This amounts to the IRS' channel gain being between $41 \%$ and $99 \%$ of the direct channel gain. Hence, the channel over the IRS not only results in higher SNR but also provides resilience against visibility outages. This comes at the cost of a delay spread that ranges from $3.0215 \mu$ s to $3.3385 \mu$ s during the satellite pass, which corresponds to 6043 to 6677 periods of the carrier signal. This indicates a minimum cyclic prefix $(\mathrm{CP})$ below $4 \mu$ for orthogonal frequency-division multiplexing (OFDM) transmission whereas $5 \mathrm{G}$ supports a maximum CP of $4.7 \mu$ s [16]. Observe that the transmission delay is already incorporated in the system model and that the results only depend on the delay spread. However, rotating a planar reflector in the same way does not result in a noticeable gain over the results in Figs. 3 and 4. Thus, the observed gains are due to the combination of rotating the IRS and configuring it with the Pareto optimal phase shifts from Theorem 1.

\section{CONCLUSiOns}

We have developed a continuous time model for IRS-assisted LOS communication with a moving receiver. The analysis includes a careful consideration of the time delays. We have

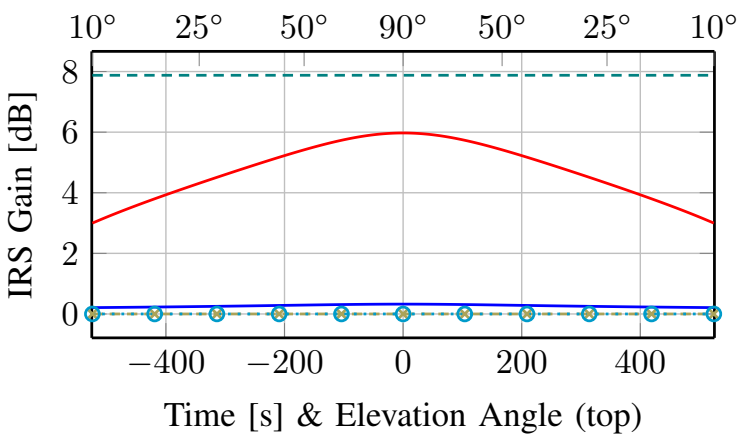

Fig. 4. Gain of using IRS over LOS only communication (legend as in Fig. 3).

shown that the phase shifts can be chosen such that they maximize the received power without incurring any Doppler spread. Moreover, they can be kept within the interval $[0,2 \pi]$ which is in the feasible range of recent IRS prototypes [7]. It also results in the minimum delay spread under meaningful IRS operation. In a numerical study, we demonstrate the benefits of IRS-assisted LEO satellite communication and show that the SNR is increased by $3 \mathrm{~dB}$ to $6 \mathrm{~dB}$ for an IRS the size of two billboards. This requires rotating the IRS such that it has a favorable orientation to the transmitter and receiver. Finding this optimal orientation is left open for future work. Other open topics are the inclusion of statistical channel model to account for atmospheric effects and LOS outages.

\section{REFERENCES}

[1] C. Liaskos et al., "A new wireless communication paradigm through software-controlled metasurfaces," IEEE Commun. Mag., vol. 56, no. 9, pp. 162-169, Sep. 2018.

[2] M. Di Renzo et al., "Smart radio environments empowered by reconfigurable AI meta-surfaces: an idea whose time has come," EURASIP J. Wireless Commun. Netw., vol. 2019, no. 1, May 2019.

[3] E. Basar et al., "Wireless communications through reconfigurable intelligent surfaces," IEEE Access, vol. 7, pp. 116753-116773, 2019.

[4] Q. Wu and R. Zhang, "Towards smart and reconfigurable environment: Intelligent reflecting surface aided wireless network," IEEE Commun. Mag., vol. 58, no. 1, pp. 106-112, Jan. 2020.

[5] — , "Intelligent reflecting surface enhanced wireless network via joint active and passive beamforming," IEEE Trans. Wireless Commun., vol. 18, no. 11 , pp. 5394-5409, Nov. 2019.

[6] E. Björnson and L. Sanguinetti, "Power scaling laws and near-field behaviors of massive MIMO and intelligent reflecting surfaces," in review. [Online]. Available: https://arxiv.org/abs/2002.04960

[7] W. Tang et al., "Wireless communications with reconfigurable intelligent surface: Path loss modeling and experimental measurement," Nov. 2019. [Online]. Available: https://arxiv.org/abs/1911.05326

[8] Ö. Özdogan, E. Björnson, and E. G. Larsson, "Intelligent reflecting surfaces: Physics, propagation, and pathloss modeling," IEEE Wireless Commun. Lett., vol. 9, no. 2, pp. 581-585, May 2020.

[9] R. J. Williams, E. De Carvalho, and T. L. Marzetta, "A communication model for large intelligent surfaces," in Proc. IEEE Int. Conf. Commun Workshops (ICC Workshops), Dublin, Ireland, 62020.

[10] L. A. Zadeh, "Optimality and non-scalar-valued performance criteria," IEEE Trans. Autom. Control, vol. 8, no. 1, pp. 59-60, Jan. 1963.

[11] E. Björnson, E. A. Jorswieck, M. Debbah, and B. Ottersten, "Multiobjective signal processing optimization: The way to balance conflicting metrics in 5G systems," IEEE Signal Process. Mag., vol. 31, no. 6, pp. 14-23, Nov. 2014

[12] H. Bauer, Measure and Integration Theory. De Gruyter, 2001.

[13] K. Miettinen, Nonlinear Multiobjective Optimization. Springer, 1999.

[14] 3GPP, "Study on New Radio (NR) to support non-terrestrial networks," Tech. Rep. 38.811 V15.2.0, Sep. 2019.

[15] L. J. Ippolito, Jr., Satellite Communications Systems Engineering, 2nd ed. Wiley, 2017.

[16] 3GPP, "5G Rel-15 release description," Tech. Rep. TR 21.915 V15.0.0, Oct. 2019. 